\title{
Prediction based channel allocation performance for cognitive radio
}

\author{
S.D. Barnes*, B.T. Maharaj \\ Department of Electrical, Electronic and Computer Engineering, University of Pretoria, Pretoria, 0002, South Africa
}

\begin{abstract}
The interdependency, in a cognitive radio (CR) network, of spectrum sensing, occupancy modelling, channel switching and secondary user (SU) performance, is investigated. Achievable SU data throughput and primary user (PU) disruption rate have been examined for both theoretical test data as well as data obtained from real-world spectrum measurements done in Pretoria, South Africa. A channel switching simulator was developed to investigate SU performance, where a hidden Markov model (HMM) was employed to model and predict PU behaviour, from which proactive channel allocations could be made. Results show that CR performance may be improved if PU behaviour is accurately modelled, since accurate prediction allows the SU to make proactive channel switching decisions. It is further shown that a trade-off may exist between achievable SU throughput and average PU disruption rate. When using the prediction model, significant performance improvements, particularly under heavy traffic density conditions, of up to double the SU throughput and half the PU disruption rate were observed. Results obtained from a measurement campaign were comparable with those obtained from theoretical occupancy data, with an average similarity score of $95 \%$ for prediction accuracy, $90 \%$ for SU throughput and $70 \%$ for PU disruption rate.
\end{abstract}

Keywords:

Channel Switching, Cognitive Radio, Occupancy Modelling, Secondary User Performance, Spectrum measurements

\section{Introduction}

The way in which land becomes scarcer as urban areas become more densely populated, is readily compared to the decrease in availability of useful radio spectrum as the number of wireless devices vying for usage of similar frequency bands increases. The FCC has predicted a rapidly increasing demand for mobile data services in North America that may exceed capacity in the near future $[1,2]$. Consequently, the concept of cognitive radio (CR) has been proposed [3] as an attempt at making more efficient usage of the radio frequency spectrum through opportunistic spectrum access.

Performance in CR networks is inherently affected by the spectrum sensing (SS) and channel selection process. Incorrect identification of spectrum opportunities and furthermore, subsequent suboptimal channel selections, could result in unnecessary delays. These delays may lead to a degradation in secondary user (SU) performance and may also bring about unnecessary interference for existing primary users (PU). Accurate, proactive channel occupancy predictions should improve the channel allocation process by helping to minimise the probability of incorrectly detecting spectrum holes, thus actively reducing interference delays and transmission gaps due to suboptimal channel switching [4].

In [5], a two-state Hidden Markov model (HMM), which is used to predict PU behaviour, is proposed. This model relies on historical usage data. In [6], usage of historical PU behaviour,

\footnotetext{
${ }^{*}$ Corresponding author, Tel.: +27 124202872.

Email address: simonbarnes@ieee.org (S.D. Barnes)
}

measured from the $6 \mathrm{GHz}$ region, was shown to improve the SS decision making process. Furthermore, intelligent approaches to channel switching, based on proactive channel predictions, were proposed in [4] and [7].

In this paper, the work in [4] and [7] has been extended by using the HMM in [5], to investigate the effect that intelligent channel switching has on SU performance in a CR network. The interdependent relationship between spectrum sensing, PU traffic modelling, channel switching, SU throughput and average PU disruption rate, is the main focus of the article.

Firstly, prediction accuracy using the HMM is investigated. The HMM was chosen, since it allows for the assumption that PU traffic can be independent of a specific statistical distribution (PU traffic was assumed to follow an exponential distribution in [4] and [7]).

Secondly, the authors present a proactive channel switching simulator for performing simulations to investigate the effect that the channel occupancy model has on the channel switching process and the subsequent effect that this has on SU data throughput and PU disruption rate. To the author's knowledge, this is currently a unique contribution to the field of a CR.

Practical spectrum measurements of GSM traffic were used to verify the results obtained by theoretical simulation. Since there appeared to be a lack of information surrounding the selection of a signal detection threshold for the measured data, a novel approach to calculating this was proposed by the authors. 


\section{Channel occupancy}

Before SU communication may be initiated, it is imperative that the SU is able to determine which channels are already occupied by PUs. Furthermore, the ability of the SU to predict future PU behaviour may provide significant benefits to both sets of users.

\subsection{Hidden Markov occupancy model}

It is necessary to model and predict channel occupancy since enabling the SU to predict future PU behaviour allows for potential improvement to the channel allocation process. One of the methods suggested for modelling channel occupancy in CR networks is the two-state HMM [5, 8, 9], where sub-band spectrum occupancy is modelled as a consecutive sequence of binary states that represent sub-band channel occupancy (the exponential distribution is an alternative approach explored in the literature [10]). HMMs are suited to this problem since true occupancy states are not always known to SUs after the SS process.

\subsubsection{HMM structure}

A HMM comprises a set $\boldsymbol{Z}$ of $M$ possible states,

$$
\boldsymbol{Z}=\left\{z_{1}, z_{2}, \cdots, z_{M}\right\}
$$

and a set $\boldsymbol{X}$ of $N$ possible emissions,

$$
\boldsymbol{X}=\left\{x_{1}, x_{2}, \cdots, x_{N}\right\}
$$

Two statistical parameters govern the operation of a HMM. The first parameter $\boldsymbol{P}$ is an $M$-by- $M$ state transition probability matrix representing the probabilities associated with changing from one state to another [11] and is given by,

$$
\boldsymbol{P}=\left(p_{i j}\right)_{M \times M} \quad i, j \in \boldsymbol{Z},
$$

where the individual elements of (3) are denoted by,

$$
p_{i j}=P_{r}\left(z_{n}=j \mid z_{n-1}=i\right) \quad 2 \leq n \leq L,
$$

and $L$ represents the length of an observation period.

Similarly, the second parameter $\boldsymbol{E}$ is an $M$-by- $N$ emission probability matrix that represents the probabilities associated with obtaining a certain output given that the model is currently in a true state $z_{n}$, and is given by,

$$
\boldsymbol{E}=\left(e_{i h}\right)_{M \times N} \quad i, h \in \boldsymbol{X},
$$

where the individual elements of (5) are denoted by,

$$
e_{i}(h)=P_{r}\left(x_{n}=h \mid z_{n}=i\right) \quad 2 \leq n \leq \tau .
$$

HMM parameters may be denoted by $\lambda=(\boldsymbol{P}, \boldsymbol{E}, \pi)$, where $\pi$ is the initial state distribution.

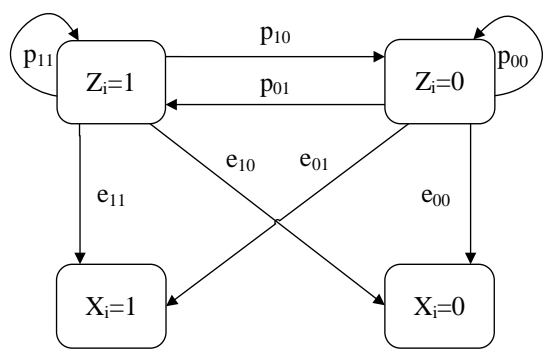

Figure 1: HMM representation of binary sub-band spectrum occupancy.

\subsubsection{Traffic occupancy model}

Proposed in $[5,12]$, the model illustrated in Fig. 1 is a two dimensional HMM with state space $\boldsymbol{Z}=\{0,1\}$ and emission state space $\boldsymbol{X}=\{0,1\}$.

At any given time, the channel may be detected to be either occupied by a PU, $Z_{i}=1$ or available for use by a SU $Z_{i}=0$. Since the HMM is used to predict a sequence of channel occupancy states, both $P(\boldsymbol{X} \mid \boldsymbol{\lambda})$ and the HMM model parameters need to be determined. Initially, an educated guess is made for these parameters. A set of observations $\boldsymbol{O}(t)=\left\{x_{t-1}, x_{t-2}, \cdots, x_{t-L}\right\}$ are then collected, by observing the band of interest for a time interval comprising a maximum of $L$ observations. The model parameters are then fine-tuned by feeding these observations into a model training algorithm. After training has been performed, the model is used to predict the most likely sequence of near-future channel occupancy states $\widehat{\boldsymbol{Z}}=\left\{z_{t+1}, z_{t+2}, \cdots, z_{N}\right\}$. Based on these predictions, the SU may now be allocated a channel within which to operate.

\subsection{Model training algorithms}

The accuracy level that the model is able to achieve is influenced by the accuracy of its parameters, i.e. if the model is poorly trained then it will not accurately represent the statistics of the channel under investigation. For comparison, two different model training algorithms are selected: a Viterbi based algorithm (VBA) and the Baum-Welch algorithm (BWA).

\subsubsection{Viterbi based algorithm}

A VBA for training HMMs is described in [13]. Given $\boldsymbol{O}(t)$ and an estimate for $\lambda$, the Viterbi algorithm may be used to calculate the most probable sequence of hidden states by recursively selecting the path with the highest probability $\pi^{*}$, such that,

$$
\pi^{*}=\arg \max _{\pi \in \mathbf{Z}} P(x, \pi) .
$$

If $V_{t, k}$ is the most probable path ending in state $k$, with $V_{0,0}=1$, then these probabilities can be obtained as follows,

$$
V_{t, k}=\log \left[P\left(x_{t} \mid k\right)\right]+\max _{z \in Z}\left[\log \left(p_{z, k}\right)+V_{t-1, z}\right] .
$$

Using $\boldsymbol{O}(t)$ and $\pi^{*}$, the model parameters are then re-estimated by counting all particular transitions and emissions that occur, according to the following expressions[13],

$$
p_{k l}=\frac{P_{k l}}{\sum_{a=0}^{M-1} P_{k a}} \quad \text { and } \quad e_{k}(b)=\frac{E_{k}(b)}{\sum_{c=0}^{N-1} E_{k}(c)},
$$


where $P_{k l}$ is the number of transitions from state $k$ to state $l$ and $E_{k}(b)$ the number of emissions of $b$ from state $k$. A value for $\lambda$ is then found that maximises the contribution to the log likelihood of the observation sequence, $\log P\left[x_{t-1}, \cdots, x_{t-L} \mid \lambda, \pi^{*}\left(x_{t-1}\right), \cdots, \pi^{*}\left(x_{t-L}\right)\right]$.

This process is repeated until the difference between consecutive values of this likelihood fall below a predefined threshold (chosen as 0.001 in this article).

\subsubsection{Baum-Welch algorithm}

The BWA is an instance of a generalised expectationmaximisation (EM) algorithm and makes use of both the forward and backward algorithms [11]. If the number of hidden and visible states is known, then it is possible to obtain a good solution for $\lambda$ by iteratively updating the weights of the BWA until certain convergence criteria have been met. The forward variable $\alpha_{\tau}$ is the probability of obtaining the partial observation sequence, $\boldsymbol{O}$, when it terminates in state $i$, given as,

$$
\alpha_{\tau}(i)=p\left(x_{1}, x_{2}, \cdots, x_{t}, z_{t}=i \mid \lambda\right),
$$

and forms part of a recursive relationship known as the forward algorithm,

$$
\alpha_{t+1}(j)=e_{j}\left(x_{t+1}\right) \gamma_{t} \quad 1 \leq j \leq M, 1 \leq t \leq L-1,
$$

where $\gamma_{t}$ is given by,

$$
\gamma_{t}=\sum_{i=1}^{M} \alpha_{t}(i) p_{i j} \quad 1 \leq j \leq M, 1 \leq t \leq L-1,
$$

and the first instance of the forward variable is,

$$
\alpha_{t+1}(j)=\pi_{j} e_{j}\left(x_{1}\right) \quad 1 \leq j \leq M .
$$

Therefore,

$$
P(\boldsymbol{X} \mid \lambda)=\sum_{i=1}^{M} \alpha_{L}(i)
$$

\section{Channel switching simulator}

A software simulator has been developed to investigate the effects that channel switching may have on the performance of SUs while operating within a CR network.

\subsection{Physical layer considerations}

The channel switching simulator has been set up to operate under various physical layer parameters that are based on the IEEE 802.22 wireless regional area network standard (WRAN) for CR [14]. The standard specifies a number of adaptive modulation $\Theta$ and coding rate $r$ modes. The authors have chosen to perform simulations using mode 3 . This mode specifies a spectral efficiency of $0.76 \mathrm{~b} / \mathrm{s} / \mathrm{hz}$ incorporating quadrature phaseshift keying (QPSK) with a $d=1 / 2$ rate forward error correction (FEC) code.

Guidelines pertaining to $\mathrm{CR}$ frame structure are provided for by the media access control (MAC) layer specification of the
Table 1: Physical layer parameters for investigating SU performance.

\begin{tabular}{|c|ccccc|}
\hline Param. & $\Theta$ & $d$ & $B W$ & $t_{f}$ & $\rho$ \\
\hline Value & QPSK & $1 / 2$ & $100 \mathrm{kHz}$ & $100 \mathrm{~ms}$ & 5 \\
\hline
\end{tabular}

standard. The standard suggests that data should be transmitted in $t_{s f}=16 t_{f}$ sized superframes. Where $t_{f}=10 \mathrm{~ms}$ is the frame period.

Due to physical limitations on the speed at which spectrum measurements were able to be taken, the frame period had to be adjusted to be ten times longer than that specified in the standard. Thus the frame period utilised was $t_{f}=100 \mathrm{~ms}$. Although $t_{f}$ was much longer than recommended by the standard, it was assumed that this would not unduly influence the results obtained, since the average length of a mobile telephone call has been reported at around 101s [15] (when $L=400$, observation time would be 40s). Since the bandwidth of a GSM channel is $200 \mathrm{KHz}$ [16], a channel bandwidth of $B W=100 \mathrm{KHz}$ was chosen. For the purpose of channel switching analysis another MAC structure was added: the superblock. A superblock consists of $t_{s b}=32 t_{s f}$ superframes. These physical layer considerations are summarised in Table 1.

\subsection{Channel-switching algorithm}

The channel switching simulator makes use of the channel occupancy and prediction model, described in Section 2, to investigate the performance of a single $\mathrm{SU}$ in a $\mathrm{CR}$ network. A flow diagram of the simulator is presented in Fig. 2.

To begin with, the SU must perform wide-band spectrum sensing and determine the channel occupancies of a set of potential narrow-band operating channels $\boldsymbol{I}_{q}$. This is done for $L$ frame periods, such that,

$$
\boldsymbol{I}_{q}=\boldsymbol{O}_{q}(t) \quad 1 \leq q \leq \vartheta, 1 \leq t \leq L,
$$

where $\vartheta$ is the size of the channel set. $\boldsymbol{I}_{q}$ is then used to train a HMM for each channel in the set.

From these models future channel occupancy for the entire set $\boldsymbol{F}_{q}$ is then predicted,

$$
\boldsymbol{F}_{q}=\boldsymbol{X}(t) \quad t_{L} \leq t \leq \Psi,
$$

where $t_{L}$ is the frame period commencing immediately after model training has been performed and $\Psi$ is the maximum number of states that will be predicted. The foundation upon which the SU will then determine when and within which channels to operate is provided by $\boldsymbol{F}_{q}$.

Before data transmission begins, the SU will quickly sense the set of potential operating channels again for a time $t_{s n} \leq$ $t_{f}$, to test for immediate PU activity on the currently selected channel $q_{c}$. If $q_{c}$ is found to be currently available at time $t_{c}$, $\boldsymbol{O}_{q}\left(t_{c}\right)=0$, then the SU will transmit on $q_{c}$ for a time interval $t_{I}$. The interval size is governed by the selected spectrum sensing approach (discussed in Section 3.3).

However, if $q_{c}$ is found to be currently occupied, $\boldsymbol{O}_{q}\left(t_{c}\right)=1$, the SU will try to find an alternative channel to pro-actively switch to. This channel is chosen by selecting what the SU 


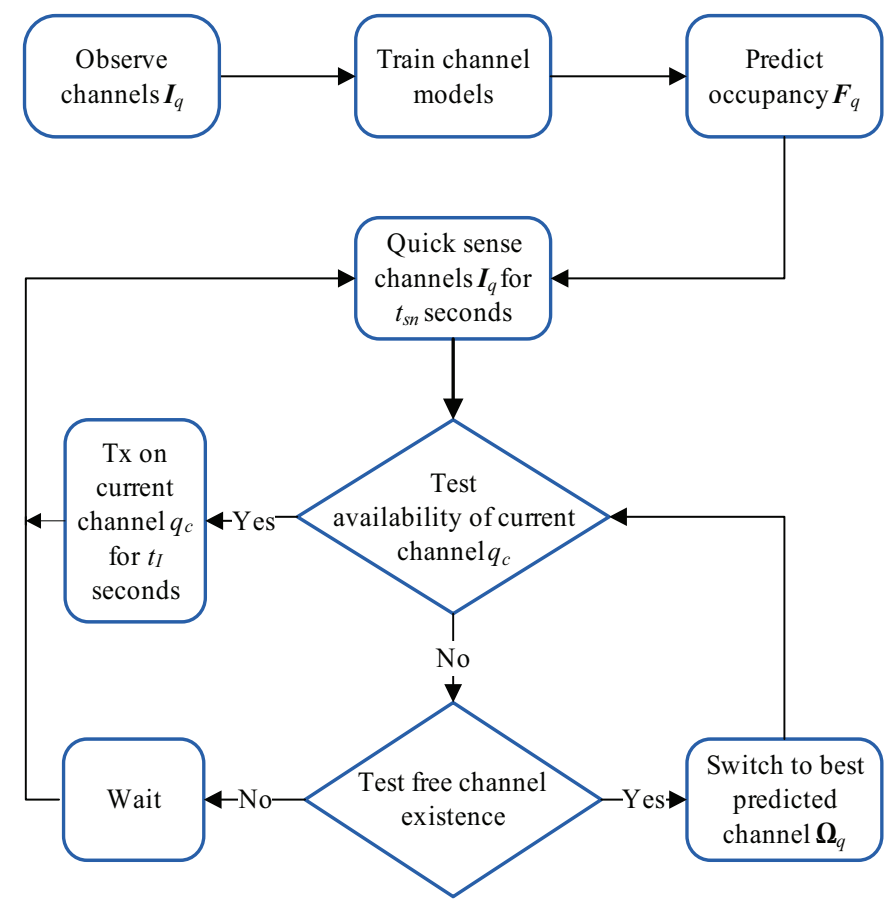

Figure 2: Algorithm for channel switching simulator.

deems to be the channel that will remain unoccupied for the longest expected number of frame periods $\Omega_{q}$ (based on future channel occupancy predictions) such that [7],

$$
\arg \max _{q} \Omega_{q} \quad t_{c} \leq \Omega_{q} t_{f} \leq t_{c}+\rho t_{f},
$$

where $\rho$ is the maximum number of future frame periods that the SU will consider when choosing $\Omega_{q}$. However, if all the channels in the set are occupied, $\boldsymbol{I}_{q}=1,1 \leq q \leq \vartheta$, then the SU will wait until $t=t_{c}+t_{I}$ before testing for an open channel again.

The SU will continue the cycle of quick sensing, testing channel availability, channel switching and data transmission until the prediction period is exceeded, i.e. $t=t_{L}+\Psi$. At this point the set of HMMs will be updated and $\boldsymbol{F}_{q}$ will be repopulated by further channel occupancy predictions, thus continuing the channel switching and transmission cycle.

\subsection{Spectrum sensing approach}

Sensing is performed to verify the occupancy status of the channel within which a SU is currently operating. This is meant to limit PU interference, however, every sensing operation does introduce added delays. The spectrum sensing approach determines how regularly the quick sensing operation is performed. A prediction dependent approach is employed which is based on the periodic sensing concept presented in [17]. However, in this paper, quick sensing relies on occupancy prediction accuracy and is only performed after $\Omega_{q}$ frame periods. The transmission interval is thus given as,

$$
t_{I}=\Omega_{q} t_{f}
$$

\section{Secondary user performance}

The criteria used to evaluate SU performance are now discussed.

\subsection{Channel occupancy model performance}

The only way that a SU will be able to perform intelligent channel switching is if it has up-to-date knowledge of all the channels that it may potentially be operating within. Amongst other factors, future PU behaviour prediction accuracy may have a significant effect on achievable CR performance. The more accurately channel occupancy is predicted, the more accurately channel allocation may be performed. Poor prediction accuracy may cause the SU to experience further delays due to additional sensing and channel switching requirements and cause PUs to be more likely to experience SU interference.

\subsection{Data throughput}

The impact that channel modelling may have on SU performance is measured by calculating the effect that channel switching accuracy has on the throughput of a single SU. Certain factors that are specific to the cognitive nature of the network will affect the actual throughput achieved. These are over and above traditional factors such as modulation scheme, coding rate and imperfect channel conditions. Any delays introduced by the CR will have a negative impact on the achievable throughput of the network. Due to the inherent nature of CR, various CR-specific delays are inevitable. These may include: sensing delays $d_{s n}$, waiting delays $d_{w t}$, switching delays $d_{s w}$, channel observation delays $d_{o b}$ and model training delays $d_{t r}$.

These delays are a function of the number of SU quick sensing operations $v_{s n}$ performed, the number of times a $\mathrm{SU}$ has to wait for a free channel to become available $v_{w t}$, the number of times a SU has to switch channels $v_{s w}$, the observation length $L$ and the number of iterations $I$ performed during the HMM training process $v_{t r}$. The sensing delay may thus be given as,

$$
d_{s n}=v_{s n} t_{s n}
$$

the waiting delay is given as,

$$
d_{w t}=v_{w t} t_{f},
$$

and the switching delay is given as,

$$
d_{s w}=v_{s w} t_{s w}
$$

where $t_{s w} \leq t_{f}$ is the time required to perform a channel switch. The channel observation delay $d_{o b}$ is given by,

$$
d_{o b}=t_{f} L
$$

and the training delay is given by,

$$
d_{t r}=v_{t r} t_{t r}
$$

where $t_{t r}<<t_{f}$ is the average time required to perform a single iteration of the HMM training algorithm. 
Table 2: Simulation parameter assumptions for CR-specific delays.

\begin{tabular}{|c|ccc|}
\hline Param. & $t_{s n}$ & $t_{s w}$ & $t_{t r}$ \\
\hline Value & $t_{f} / 2$ & $t_{f} / 2$ & $t_{f} / 10$ \\
\hline
\end{tabular}

If throughput is defined to be the rate at which data is received, then it may be presented as a function of the number of bits $k$ received over a fixed period of time $T_{s}$. Thus data throughput $R_{b}$ is given as [18],

$$
R_{b}=\frac{k}{T_{s}}
$$

The theoretically achievable throughput $R_{s u}$ based on CR factors only, i.e. the effects of modulation, coding and channel gain are ignored, may thus be expressed as follows,

$$
R_{s u}=\frac{k}{T_{c r}}
$$

where $T_{c r}$ represents the transmission time period with the additional time delay introduced by the CR process included. $T_{c r}$ is thus defined as follows,

$$
T_{c r}=T_{s}+d_{s n}+d_{w t}+d_{s w}+d_{o b}+d_{t r}
$$

A summary of the assumptions made regarding the length of these CR-specific delays, is presented in Table 2, where $t_{s n}, t_{s w}$ and $t_{t r}$ are specified as a function of $t_{f}$.

\subsection{Primary user disruption}

When a SU erroneously switches to a channel that is already occupied by a PU it will disrupt and interfere with the PU. This will occur when a channel is either incorrectly sensed and/or predicted to be unoccupied at a time when it is in actual fact not. The rate at which a SU may cause disruptions to PUs is calculated using $T_{c r}$. This is calculated as the number of times that a SU causes PU interference over a certain period of time. If $I_{c r}$ denotes the number of such events that occur during the time period $T_{c r}$, then PU disruption rate $D_{p u}$ may be expressed as,

$$
D_{p u}=\frac{I_{c r}}{T_{c r}}
$$

\section{Spectrum measurements}

Using the energy detector method, channel occupancy data was collected through a six week long spectrum measurement campaign carried out on the main campus of the University of Pretoria, South Africa [19]. The channel switching simulator (discussed in Section 3) was employed to investigate potential SU performance on this data set. For the purpose of verification, these results will be compared, in Section 6, to those obtained by theoretically based simulations.

\subsection{Spectrum sensing}

Due to its low computational and implementation complexity, the energy detection sensing technique was chosen [20]. For this technique, channel occupancy is determined by comparing the output of an energy detector to a predetermined threshold. This threshold may be heavily influenced by the environmental noise floor. The detected signal $r(n)$ may be represented by the following signal model,

$$
r(n)=s(n)+w(n),
$$

where $s(n)$ is the original PU signal and $w(n)$ denotes additive white Gaussian noise (AWGN). It is assumed that PU channel occupancy follows a binary model of being either unoccupied $H_{0}$ or occupied $H_{1}$, the outcome of a sensing operation may thus be expressed by the following binary hypothesis,

$$
\begin{aligned}
& H_{0}: r(n)=w(n), \\
& H_{1}: r(n)=s(n)+w(n) .
\end{aligned}
$$

Thus, when the PU is not present, $s(n)=0$, alternatively $s(n)>$ 0 when a PU is occupying the band.

\subsection{Occupancy threshold}

To test (29), the signal detection threshold $K_{T h}$ needs to be calculated. To do this, the received signal $r(n)$ needs to be transformed into a more detector friendly form. This may be accomplished by raising $r(n)$ to a threshold exponent $\delta$ as follows,

$$
Y=|r(n)|^{\delta} \quad 0 \leq n \leq N .
$$

In (30) $N$ is the observation vector size, while the value chosen for $\delta$ determines the extent to which smaller signal or noise components may be suppressed, or the extent to which larger components may be accentuated. To compensate for the accentuated components, $Y$ is then clipped by the sum of its mean $\mu_{s}$ and standard deviation $\sigma_{s}$. The clipped signal may be expressed as,

$$
Y_{c}=\left\{\begin{array}{rll}
Y & \text { if } & |Y| \leq \mu_{s}+\sigma_{s} \\
\mu_{s}+\sigma_{s} & \text { if } & |Y|>\mu_{s}+\sigma_{s} .
\end{array}\right.
$$

The signal detection threshold $K_{T h}$ may now be calculated by adding the mean of the clipped signal $\mu_{c}$ to a preselected constant $\gamma$, and is thus given as,

$$
K_{T h}=\mu_{c}+\gamma
$$

The design choices for $\delta$ and $\gamma$ affect channel occupancy accuracy. Since these parameters have a direct influence on $K_{T h}$ they need to be selected appropriately so as to minimise the probabilities associated with incorrectly detecting PU activity, namely mis-detection $P_{m d}$ and false alarm $P_{f a} . P_{m d}$ is defined as the probability of detecting a band to be free when in reality it is actually occupied. $P_{m d}$ is thus given as,

$$
P_{m d}=P_{r}\left\{Y<K_{T h}\left|H_{1}\right|\right\} .
$$

$P_{f a}$ is defined as the probability of detecting PU activity when the channel is actually free. $P_{f a}$ is given as,

$$
P_{f a}=P_{r}\left\{Y>K_{T h} \mid H_{0}\right\} .
$$



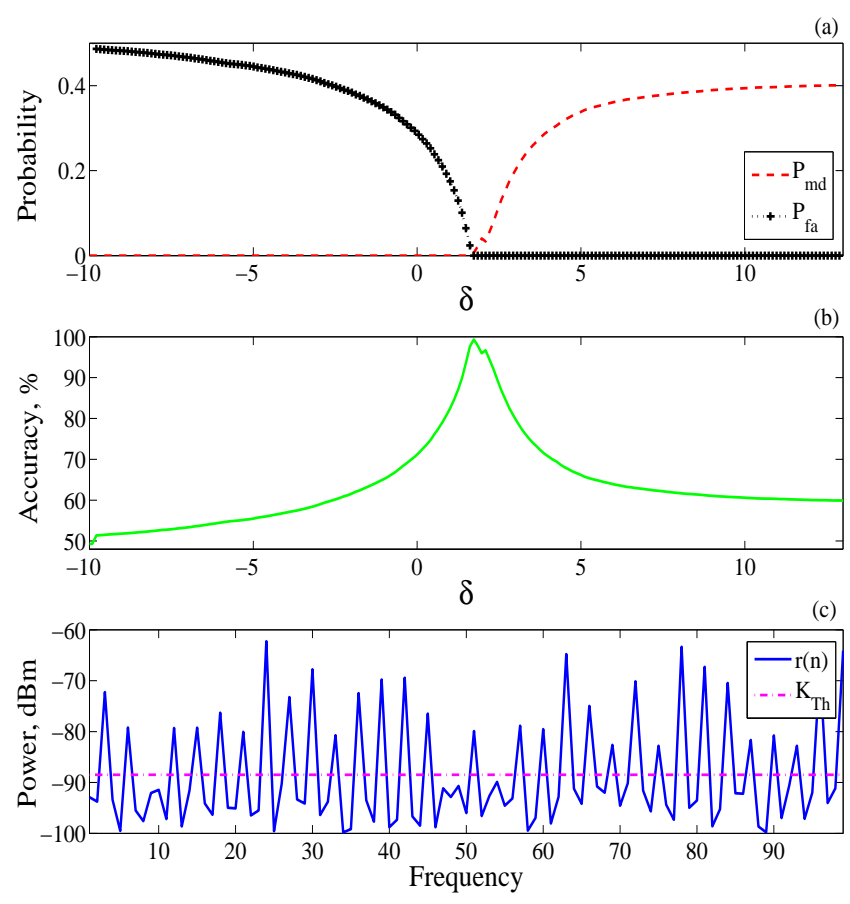

Figure 3: Simulated effect of $\delta$ on: (a) $P_{f a}, P_{m d}$, and (b) the resulting accuracy of channel occupancy detection. An illustration of $K_{T h}$ when $\delta=1.73$ is presented in part (c).

The importance of correct threshold selection is illustrated in Fig. 3, where $P_{f a}, P_{m d}$ and the resulting percentage accuracy of PU detection are compared. Fig. 3 has been generated from a set of simulated test data where transmitter information is known. The average channel occupancy of the simulated test data is $41.6 \% . P_{f a}$ and $P_{m d}$ are estimated by counting the rate at which these events occur over a test sequence of 2000 states. The calculated detection threshold, when $\delta=1.73$, is also visually illustrated. It is assumed that $\gamma=0$.

\subsection{Bands of interest}

SU performance is investigated using measured data from the South African GSM cellular bands. Occupancy data from two specific bands, presented in Table 3 , has been selected for analysis. These data sets have been chosen in an attempt to represent both high and low traffic-density conditions. Data from these bands is used to test the occupancy model and also provide inputs to the channel switching simulator (described in Section 3). Measurements have thus been taken from a portion of the GSM 900 up-link (band A) as well a portion from the GSM 1800 down-link (band B).

Table 3: Table of measured frequency bands, calculated detection thresholds and associated percentage occupancies.

\begin{tabular}{|c|c|c|}
\hline Freq. band & Band A & Band B \\
\hline Freq. (MHz) & $890.1-895.1$ & $1848.5-1853.5$ \\
Thres. (dBm) & -91.49 & -93.50 \\
Occup. (\%) & 20.47 & 84.38 \\
\hline
\end{tabular}

\subsection{Measurement system}

The system used to take spectrum measurements, based on the design in [21], employs a wide band antenna that is connected to a spectrum analyser (SA) via a low noise amplifier (LNA). Operation of the system is controlled by an automated software application that interfaces with the SA over a remote Ethernet connection. The antenna, LNA and SA are housed within an air-conditioned metal cabinet at the measurement site.

\subsection{Measurement description}

The measurements described in this paper were taken from a $5 \mathrm{MHz}$ block of spectrum, spaced at a channel frequency interval of $100 \mathrm{kHz}$. Each measured channel consisted of a sweep of 512 time samples that were spaced $100 \mathrm{~ms}$ apart from each other. Thus, each band was represented by a frequency-time matrix of 50 frequency bins of 512 time samples each $(5 \mathrm{MHz}$ x $51.2 \mathrm{~s}$ ). Measurements were taken every two hours during a measurement campaign that covered a period of six weeks.

\subsection{Measured channel occupancy}

The measured average frequency-time power spectra of the bands presented in Table 3 are illustrated in Fig. 5 and Fig. 4 respectively.

Detection thresholds for these bands have been calculated according to the procedure described in Section 5.2. The values chosen for $\delta$ in (30) were selected by a process of visual inspection. Separate values for $\delta$ were selected for each band due to differences in the frequency-power profile of each band (this was necessitated by the reliance of the threshold detection method on calculating mean received power levels). Threshold exponent values of $\delta=5.0$ and $\delta=0.1$ were chosen for frequency band $\mathrm{A}$ and frequency band $\mathrm{B}$ respectively.

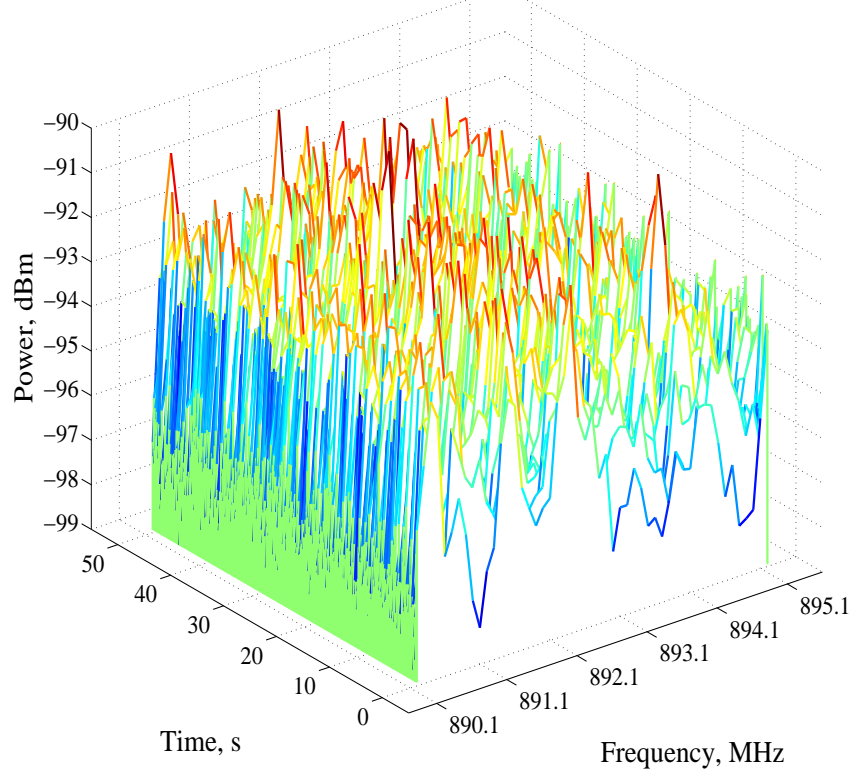

Figure 4: Measured power spectra of frequency band A. 


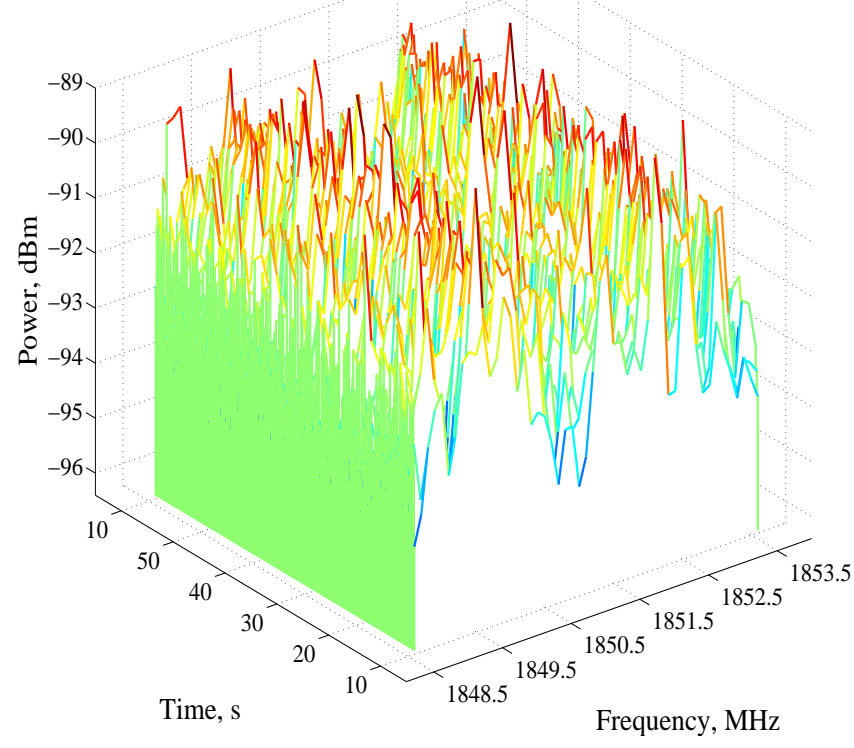

Figure 5: Measured power spectra of frequency band B.

Calculated detection thresholds and resulting percentage channel occupancies are listed in Table 3 . The calculated channel occupancy for both bands is also visually illustrated in Fig. 6 in the form of a white and black frequency-time binary occupancy map. The white areas represent PU occupancy and the black areas the absence thereof.

The selected bands clearly exhibit rapidly changing PU activity across both time and frequency. The calculated average percentage occupancy of band A is $20.47 \%$ and for band B $84.38 \%$. It is thus assumed that data from band A represents

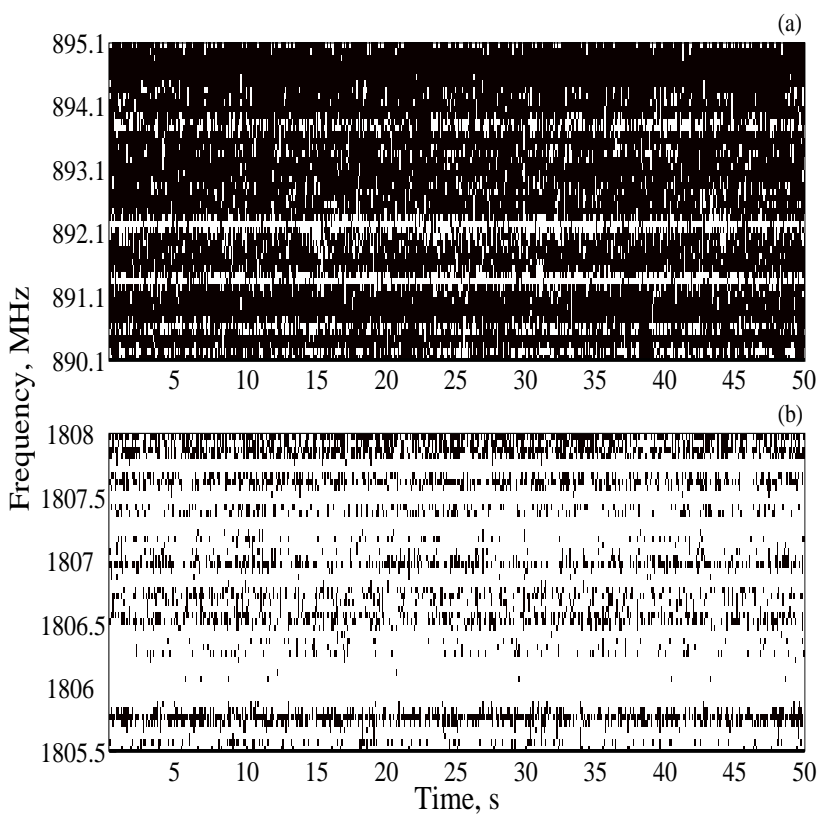

Figure 6: Binary occupancy plots for (a) frequency band A and (b) frequency band $\mathrm{B}$. practically measured low density traffic conditions and that the data from band $\mathrm{B}$ represents practically measured high density traffic conditions.

\section{Performance evaluation}

The channel switching simulator was used to investigate the accuracy of the traffic occupancy model as well as its effect on the performance of a single SU within a CR network. Simulation results were compared for both theoretical and measured channel occupancy.

\subsection{Occupancy model performance}

Performance results for the HMM based occupancy model using different training algorithms are presented in Table 4 (trained by the VBA) and Table 5 (trained by the BWA) and include results obtained from theoretical occupancy data, from measured occupancy data and the absolute difference between them $(|\Delta A|$ when comparing band $\mathrm{A}$ and $|\Delta B|$ when comparing band $\mathrm{B})$. The results include, the percentage mean prediction accuracy, the percentage standard deviation of prediction accuracy and the number of iterations required to train the model.

The performance of the model was investigated for a set of training observation lengths $L=\{200 ; 300 ; 400\}$ and it was evident that prediction accuracy improved as $L$ was increased. It was also noticeable that using the BWA to train the HMM provided a slight prediction accuracy improvement over the VBA, particularly for the measured occupancy data. However, the BWA is a more complex algorithm and was found to require many more iterations to converge on a good solution.

From Table 4 and Table 5 it is evident that for both training algorithms the results, both in terms of measured prediction accuracy and the required number of training algorithm iterations, are either comparable with or show an improvement over the simulated results (especially for shorter lengths of $L$ ). However, the model performed slightly worse when trained with the VBA in frequency band A.

\subsection{Spectrum allocation performance}

Simulations, based on both theoretical and measured occupancy data, were run on the channel switching simulator to

Table 4: Channel occupancy prediction performance using the HMM based prediction model and VBA training algorithm.

\begin{tabular}{|c|c|c|cc|cc|}
\cline { 3 - 7 } \multicolumn{1}{c|}{} & \multicolumn{2}{c|}{ Band A } & \multicolumn{2}{c|}{ Band B } \\
\cline { 2 - 7 } \multicolumn{1}{c|}{ Mean } & Theo. & Meas. & $|\% \Delta A|$ & Meas. & $|\% \Delta B|$ \\
\hline \multirow{4}{*}{ Std $\Delta$} & 200 & 75.00 & 72.45 & 3.40 & 82.54 & 10.05 \\
& 300 & 84.57 & 81.81 & 3.26 & 86.60 & 2.40 \\
& 400 & 92.70 & 89.85 & 3.07 & 92.79 & 0.10 \\
\hline \multirow{4}{*}{ Iterat. } & 200 & 11.31 & 1.56 & 86.21 & 1.69 & 85.06 \\
& 300 & 6.43 & 1.35 & 79.00 & 1.96 & 69.52 \\
& 400 & 3.82 & 0.52 & 86.39 & 0.01 & 99.74 \\
\hline & 200 & 4.0 & 5.49 & 37.25 & 4.80 & 20.00 \\
& 300 & 4.0 & 15.14 & 278.5 & 4.98 & 24.50 \\
& 400 & 4.0 & 5.65 & 41.25 & 5.16 & 29.00 \\
\hline
\end{tabular}


Table 5: Channel occupancy prediction performance using the HMM based prediction model and BWA training algorithm.

\begin{tabular}{|c|c|c|c|c|c|c|}
\hline & \multirow[b]{2}{*}{$\mathbf{L}$} & \multirow[b]{2}{*}{ Theo. } & \multicolumn{2}{|c|}{ Band A } & \multicolumn{2}{|c|}{ Band B } \\
\hline & & & Meas. & $\overline{|\overline{\mid}| \% \Delta A \mid}$ & Meas. & $\overline{|\overline{\mid \%} \Delta B|}$ \\
\hline \multirow{3}{*}{ Mean } & 200 & 76.49 & 81.99 & 7.19 & 85.72 & 12.07 \\
\hline & 300 & 84.06 & 87.44 & 4.02 & 89.78 & 6.80 \\
\hline & 400 & 92.51 & 92.57 & 0.06 & 92.59 & 0.09 \\
\hline \multirow{3}{*}{ Std } & 200 & 8.62 & 1.78 & 79.35 & 2.15 & 75.06 \\
\hline & 300 & 6.28 & 1.19 & 81.05 & 1.01 & 83.92 \\
\hline & 400 & 3.33 & 0.57 & 82.88 & 0.99 & 70.27 \\
\hline \multirow{3}{*}{ Iterat. } & 200 & 106.8 & 77.35 & 27.57 & 101.2 & 5.24 \\
\hline & 300 & 116.6 & 79.37 & 31.93 & 91.84 & 21.23 \\
\hline & 400 & 126.5 & 90.43 & 28.51 & 90.48 & 28.47 \\
\hline
\end{tabular}

investigate the effect that prediction model based channel allocation had on potentially achievable SU throughput and PU disruption rate.

\subsubsection{Simulation parameters and assumptions}

An HMM was used to generate test data for both a heavy and a light density traffic scenario. To do this, parameters were chosen as $\boldsymbol{P}=\boldsymbol{P}_{t}$ for heavy density traffic (78.75\% occupancy), and $\boldsymbol{P}=1-\boldsymbol{P}_{t}$ for light density traffic (31.84\% occupancy). For both scenarios, $\boldsymbol{E}=\left|\boldsymbol{I}_{2}-0.05\right|$, where $\boldsymbol{I}_{2}$ is a two-dimensional identity matrix and the transition matrix $\boldsymbol{P}_{t}$ was defined as [5],

$$
\boldsymbol{P}_{t}=\left[\begin{array}{ll}
0.30 & 0.70 \\
0.20 & 0.80
\end{array}\right]
$$

Initial parameter guesses, for training the prediction model, were chosen as $\boldsymbol{P}=\boldsymbol{P}_{t}-0.3 \boldsymbol{I}_{2}+0.15$ and $\boldsymbol{E}=\left|\boldsymbol{I}_{2}-0.09\right|$. A summary of parameter choices is provided in Table 6 .

The VBA was chosen to train the occupancy model (due to its lower computational complexity) and the observation and training window was limited to $L+\Psi=512$ consecutive states. This meant that as $L$ was increased, $\Psi$ was decreased by a proportionate amount.

The physical layer parameters listed in Table 1 were adhered to and simulations were run for a length of one superblock $t_{s b}$ for $S_{x}=1024 t_{s b}$ iterations.

Since a-priori information about PU activity is not known to SUs, channel occupancy was determined by selecting a detection threshold at acceptable SNR conditions. Therefore, only CR-specific effects were considered, i.e. the effects of channel gain were ignored.

\subsubsection{Simulator results}

Results for SU throughput and PU disruption rate, based on theoretical occupancy data, are presented in Fig. 7 and Fig. 8 respectively, and the equivalent results for frequency band $\mathrm{A}$

Table 6: Model parameters for simulating traffic density.

\begin{tabular}{|c|c|c|c|}
\hline & Initial guess & Light traffic & Heavy traffic \\
\hline $\boldsymbol{P}$ & $\boldsymbol{P}_{t}-0.3 \boldsymbol{I}_{2}+0.15$ & $1-\boldsymbol{P}_{t}$ & $\boldsymbol{P}_{t}$ \\
$\boldsymbol{E}$ & $\left|\boldsymbol{I}_{2}-0.09\right|$ & $\left|\boldsymbol{I}_{2}-0.05\right|$ & $\left|\boldsymbol{I}_{2}-0.05\right|$ \\
\hline
\end{tabular}

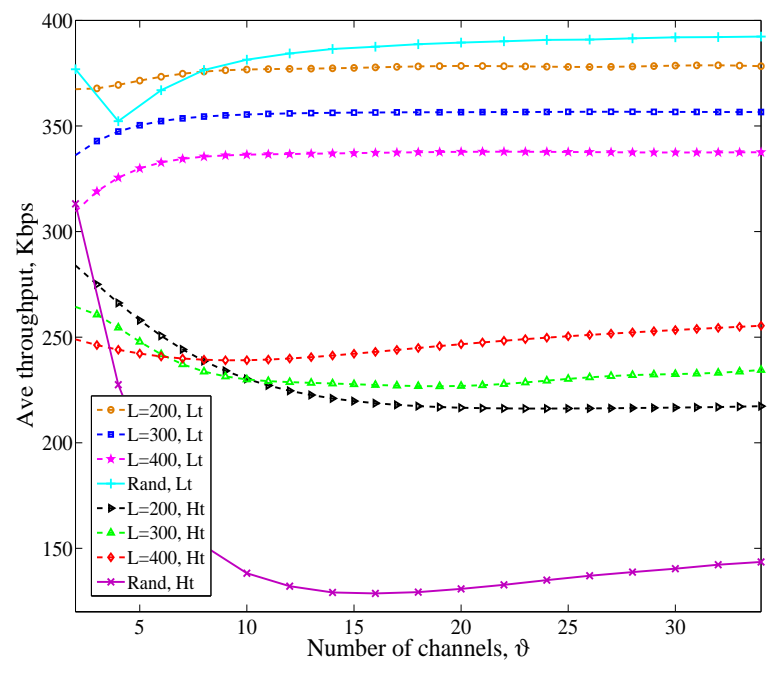

Figure 7: Average SU throughput calculated from theoretical occupancy data.

and frequency band B are presented in Fig. 9 and Fig. 10. Results based on prediction are also compared against those where channel switching is performed on a random basis. For comparative purposes, the random approach to switching was chosen to illustrate the benefits that channel occupancy prediction may have on CR network performance.

Since prediction accuracy improves as $L$ increases, a longer $L$ should mean that fewer channel switching operations will be required of the SU. This was shown to be true for heavy traffic, since throughput increased and PU disruption decreased as both $L$ and $\vartheta$ were increased (throughput doubled and PU disruption halved compared to random switching when $L=400$ and $\vartheta=$ 20). This trend was reversed for throughput under light traffic (throughput decreased by between $15 \%$ to $30 \%$ when $L=400$ and $\vartheta=20)$.

A possible explanation is that, under light traffic density conditions, there are many more channels available for the SU to switch to and thus in terms of throughput, the reduction gained

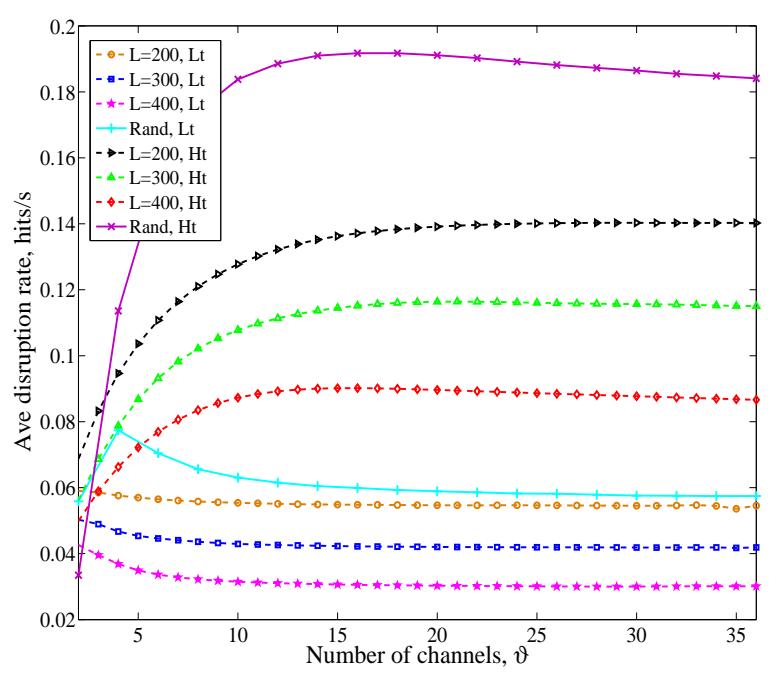

Figure 8: Average PU disruption rate calculated from theoretical data. 


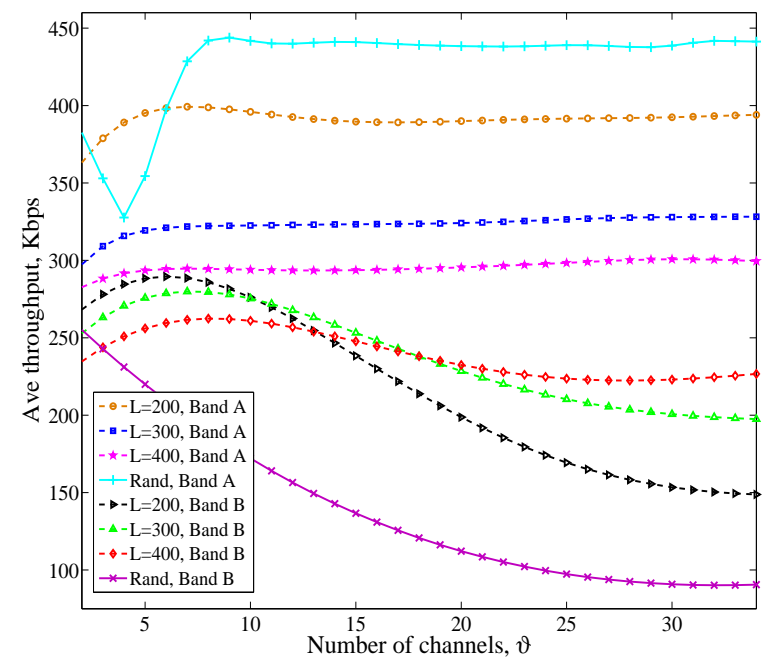

Figure 9: Average SU throughput calculated from measured occupancy data.

for $v_{s w}$ is too small to justify the time spent on gathering training sequence observations, i.e., more time is spent on training the model than is saved by the achieved reduction in $v_{s w}$.

As expected, heavy traffic density had a noticeably negative effect on SU throughput and even more so on the PU disruption rate when compared to light traffic density (approximately a $25 \%$ reduction in throughput and a $250 \%$ increase in the PU disruption rate when $L=400$ and $\vartheta=20$ ).

\subsection{Similarity}

To test how results based on theoretical occupancy $\boldsymbol{H}$ compared to those based on measured occupancy $\boldsymbol{\Gamma}$, an average percentage similarity score $\eta$ was calculated as follows,

$$
\eta=100\left[1-\left(\frac{|\boldsymbol{\Gamma}-\boldsymbol{H}|}{\boldsymbol{H}}\right)\right] .
$$

Average percentage similarity scores, listed in Table 7, indicate good correlation between theoretical and measured results for

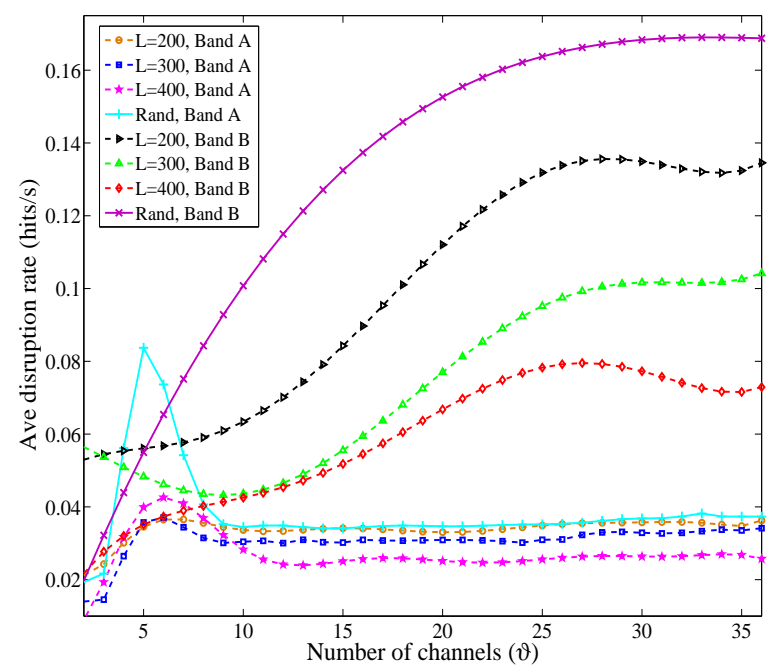

Figure 10: Average PU disruption rate calculated from measured data.
Table 7: Similarity scores for Frequency bands A and B as compared to results obtained from theoretical occupancy data.

\begin{tabular}{|c|ccc|ccc|}
\hline & \multicolumn{3}{|c|}{ Freq. band A } & \multicolumn{3}{c|}{ Freq. band B } \\
\hline VBA & 96.6 & 96.7 & 96.9 & 90.0 & 97.6 & 99.9 \\
BWA & 92.8 & 96.0 & 99.9 & 87.9 & 93.2 & 99.9 \\
$\boldsymbol{R}_{\boldsymbol{s} u}$ & 96.0 & 91.1 & 88.3 & 82.4 & 88.7 & 92.2 \\
$\boldsymbol{D}_{\boldsymbol{p} \boldsymbol{u}}$ & 61.4 & 72.1 & 77.5 & 75.6 & 67.9 & 68.6 \\
\hline $\mathbf{L}$ & 200 & 300 & 400 & 200 & 300 & 400 \\
\hline
\end{tabular}

both prediction accuracy $(\eta \approx 95 \%)$ and SU throughput $(\eta \approx$ $90 \%)$. There is a lower but still fair correlation $(\eta \approx 70 \%)$ for PU disruption rate. In general, lower values of $\eta$ may be attributed to variations in PU occupancy patterns. However, significant performance improvements for PU disruption, based on measured occupancy, were responsible for the lower $\eta$ values for PU disruption.

\section{Conclusion}

In this article, the interdependency of spectrum sensing, PU traffic modelling, channel switching and SU performance, was investigated for a CR network scenario. A channel switching simulator was presented and a channel switching algorithm, based on a two-state HMM, was proposed. SU throughput and PU disruption rate were compared under both heavy and light traffic density conditions for both simulated and practically measured occupancy data. Results obtained by the proposed channel switching and allocation method indicated significant SU throughput gains (up to $200 \%$ increase), particularly under heavy traffic conditions, and an overall reduction in the PU disruption rate (up to $50 \%$ reduction) under both light and heavy traffic density conditions. SU performance was degraded by high traffic density but the proposed channel switching algorithm helped to limit the severity of degradation. Results obtained from measured occupancy data were comparable with those obtained through simulation. From the work presented it may be concluded that the proposed method is accurate, as corroborated by the results obtained from the measured occupancy data.

\section{Acknowledgement}

This research is supported by the Sentech Chair in Broadband Wireless Multimedia Communication (BWMC), the National Research Foundation (NRF) and the Independent Communications Authority of South Africa (ICASA).

\section{References}

[1] Federal Communications Commission, Mobile broadband: the benefits of additional spectrum, FCC staff technical paper (Oct. 2010).

[2] Federal Communications Commission, Spectrum policy task force report, Tech. Rep. ET Docket 02-155 (Nov. 2002).

[3] J. Mitola III, G. Q. Maguire Jr., Cognitive radio: making software radios more personal, IEEE Pers. Commun. 6 (4) (1999) 13-18.

[4] L. Yang, L. Cao, H. Zheng, Proactive channel access in dynamic spectrum networks, Physical Commun. 1 (2) (2008) 103-111. 
[5] C. Ghosh, C. Cordeiro, D. P. Agrawal, M. B. Rao, Markov chain existence and hidden markov models in spectrum sensing, in: Proc. 7th Annu. IEEE Int. Conf. Pervasive Comput. Commun., Galveston, TX, 2009, pp. 1-6.

[6] M. Wellens, A. de Baynast, P. Mähönen, Performance of dynamic spectrum access based on spectrum occupancy statistics, IET Commun. 2 (6) (2008) 772-782.

[7] M. Höyhtyä, S. Pollin, A. Mämmelä, Performance improvement with predictive channel selection for cognitive radios, in: Proc. 1st Int. Workshop Cognitive Radio Adv. Spectr. Manage., Aalborg, Denmark, 2008, pp. 1-5.

[8] S. Geirhofer, L. Tong, B. M. Sadler, A measurement-based model for dynamic spectrum access in WLAN channels, in: Proc. IEEE Military Commun. Conf., Washington, DC, 2007, pp. 1-7.

[9] Z.-J. Zhao, S.-L. Zheng, C.-Y. Xu, X.-Z. Kong, Discrete channel modelling based on genetic algorithm and simulated annealing for training hidden Markov model, Chinese Physics 16 (6) (2007) 1619-1623.

[10] C. Ghosh, S. Pagadarai, D. P. Agrawal, A. M. Wyglinski, A framework for Statistical Wireless Spectrum Occupancy Modeling, IEEE Trans. Wireless Commun. 9 (1) (2010) 38-44.

[11] R. O. Duda, P. E. Hart, D. G. Stork, Pattern classification, 2nd Edition, Wiley \& Sons, Inc., 2001.

[12] S. D. Barnes, B. T. Maharaj, Performance of a hidden Markov channel occupancy model for cognitive radio, in: Proc. IEEE AFRICON Conf., Livingstone, Zambia, 2011, pp. 1-6.

[13] R. Durbin, S. Eddy, A. Krogh, G. Mitchison, Biological sequence analysis: probabilistic models of proteins and nucleic acids, Cambridge University Press, 1998.

[14] C. Stevenson, G. Chouinard, L. Zhongding, H. Wendong, S. Shellhammer, W. Caldwell, IEEE 802.22: The first cognitive radio wireless regional area network standard, IEEE Commun. Mag. 47 (1) (2009) 130_ 138.

[15] L. A. Grzybowski, P. Pereira, The consumer loss of the minimum duration for mobile telephone calls, Telecommun. Policy 33 (3) (2009) 200-206.

[16] T. S. Rappaport, Wireless communications: principles and practice, 2nd Edition, Prentice Hall, 2002.

[17] P. Paysarvi-Hoseini, N. C. Beaulieu, Optimal wideband spectrum sensing framework for cognitive radio systems, IEEE Trans. Signal Process. 59 (3) (2011) 1170-1182.

[18] J. G. Proakis, M. Salehi, Digital communications, 5th Edition, McGraw Hill, Singapore, 2008

[19] S. D. Barnes, Cognitive radio performance optimisation through spectrum availability prediction, Master's thesis, University of Pretoria (2012).

[20] T. Yücek, H. Arslan, A survey of spectrum sensing algorithms for cognitive radio applications, IEEE Commun. Surveys Tuts. 11 (1) (2009) 116130.

[21] M. Wellens, J. Wu, P. Mähönen, Lessons learned from an extensive occupancy measurement campaign and stochastic duty cycle, Mobile Netw. Appl. 15 (3) (2010) 461-474.

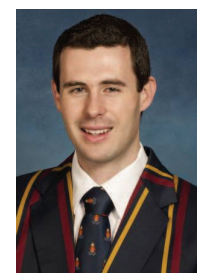

Simon Barnes received his MEng in Electronic Engineering from the University of Pretoria. $\mathrm{He}$ is currently based at the Sentech Chair in Broadband Wireless Multimedia Communications in the Department of Electrical, Electronic and Computer Engineering at the University of Pretoria. His research interests include cognitive radio, dynamic spectrum access and spectrum utilisation.

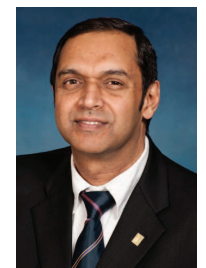

Sunil Maharaj received his PhD in Wireless Communications from the University of Pretoria. Professor Maharaj currently holds the position of Sentech Chair in Broadband Wireless Multimedia Communications in the Department of Electrical, Electronic and Computer Engineering at the University of Pretoria. His research interests are in MIMO channel modelling, OFDM-
MIMO systems and cognitive radio.

\section{Appendix A. List of symbols}

A list of the most important symbols used in this article is provided in Table A.8.

Table A.8: List of symbols used.

\begin{tabular}{|c|c|}
\hline Symbol & Definition \\
\hline$Z$ & Matrix of possible states \\
\hline$M$ & Number of possible states \\
\hline $\boldsymbol{X}$ & Matrix of possible emissions \\
\hline$N$ & Number of possible emissions \\
\hline $\boldsymbol{P}$ & State transition probability matrix \\
\hline $\boldsymbol{E}$ & Emission probability matrix \\
\hline $\boldsymbol{O}$ & Set of channel observations \\
\hline$L$ & Observation length \\
\hline$\pi$ & Initial state distribution \\
\hline $\boldsymbol{I}_{q}$ & Potential operating channel set \\
\hline $\boldsymbol{F}_{q}$ & Predicted operating channel set \\
\hline$\Psi$ & Maximum number of predicted states \\
\hline $\mathbf{\Omega}_{q}$ & Maximum expected free channel time \\
\hline$\rho$ & Maximum number of future frames \\
\hline$B W$ & Channel bandwidth \\
\hline$\Theta$ & Modulation scheme \\
\hline$d$ & Coding rate \\
\hline$q_{c}$ & Currently selected channel \\
\hline$t_{I}$ & Transmission interval \\
\hline$t_{f}$ & Frame period \\
\hline$t_{s n}$ & Quick sensing time \\
\hline$d_{s n}$ & Overall quick sensing delay \\
\hline$d_{w t}$ & Waiting delay \\
\hline$t_{s w}$ & Channel switching time \\
\hline$d_{s w}$ & Overall channel switching delay \\
\hline$d_{s n}$ & Overall channel observation delay \\
\hline$t_{t r}$ & Iteration time for model training \\
\hline$d_{t r}$ & Overall model training delay \\
\hline$R_{S U}$ & Secondary user throughput \\
\hline$T_{C R}$ & Cognitive radio transmission time \\
\hline$I_{C R}$ & Number of interference events \\
\hline$D_{P U}$ & Primary user disruption rate \\
\hline$r(n)$ & Received signal \\
\hline$s(n)$ & Information component of received signal \\
\hline$w(n)$ & Noise component of received signal \\
\hline$Y$ & Noise suppressed signal \\
\hline$Y_{c}$ & Clipped signal \\
\hline$\delta$ & Noise threshold exponent \\
\hline$\mu_{c}$ & Mean of clipped signal \\
\hline$P_{m d}$ & Probability of mis-detection \\
\hline$P_{f a}$ & Probability of false alarm \\
\hline$K_{T h}$ & Detection threshold \\
\hline$\eta$ & Similarity score \\
\hline $\boldsymbol{H}$ & Theoretical occupancy matrix \\
\hline$\Gamma$ & Measured occupancy matrix \\
\hline
\end{tabular}

